\title{
Tailored accelerating beam profiles through a caustic-based approach to wavefront design
}

\author{
L. Froehly, R. Giust, F. Courvoisier, A. Mathis, M. Jacquot, L. Furfaro, J. M. Dudley
}

Département d'Optique P. M. Duffieux, Institut FEMTO-ST, UMR CNRS 6174, Université de Franche-Comté, 25030 Besançon Cedex, France

Transversally accelerating beams exhibit a curved trajectory of their point of maximum intensity. Although the well-known example of the Airy beam was introduced by Berry and Balazs over 30 years ago [1], it is only recently that they were experimentally observed [2]. This observation, however, has since generated tremendous interest, and important applications : all-optical manipulation, curved nonlinear optics and filamentation are the subject of much current research [3-5].

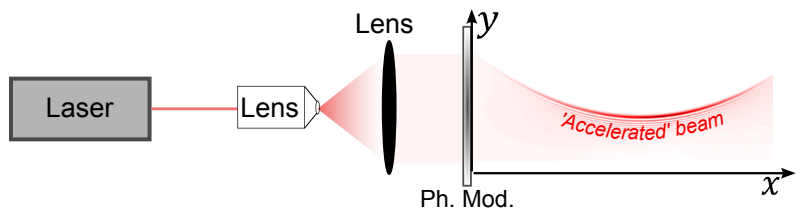

Fig. 1 Schematic of principle showing how to create an accelerating beam: Ph.Mod. is a phase modulator.

Experimentally, accelerating beam profiles are generated through the application of an appropriate phase profile upon an incident wavefront. Although the appropriate phase profiles can be calculated for the specific example of the Airy beam, the more general issue of how to design arbitrary acceleration trajectories with their appropriate phase masks is an important open problem.
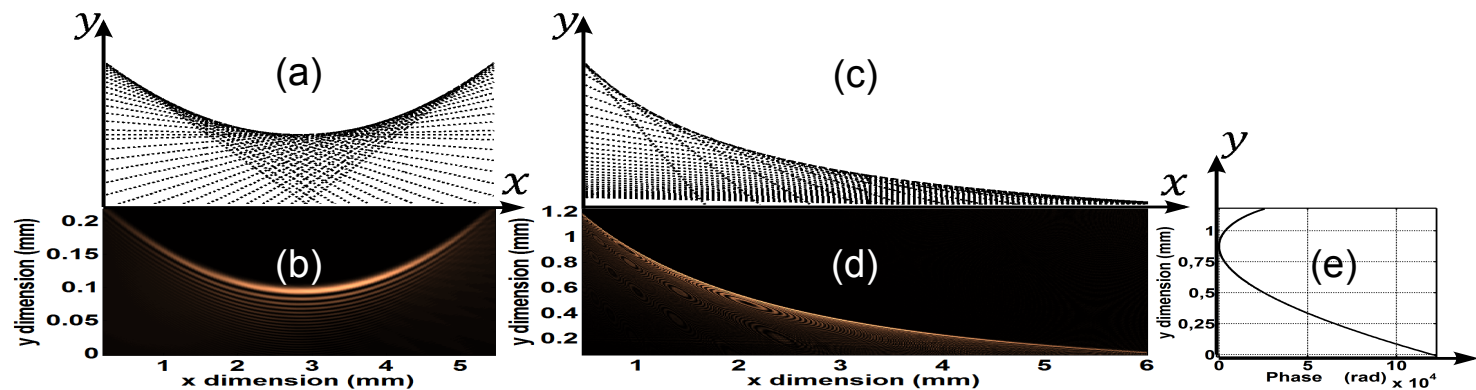

Fig. 2 Comparison between the caustic profiles and the real propagated beams:(a) Parabolic profile $y(x)$ with its generating rays. (b) is the corresponding accelerating beam obtained from propagation of the phase computed from (a).

(c) Hyperbolic profile. (d) is the corresponding accelerating beam obtained from propagation of the phase $\Phi(y)$ (Fig.2 (e)) computed from (c)

Here, we report a novel approach to generate arbitrary accelerating beam profiles. We apply an inverse-problem approach to a caustic-based description of the accelerating beam. Specifically, we consider a beam propagating along $x$ with a desired acceleration profile $y(x)$. By treating $y(x)$ as a caustic surface envelope of a family of tangential light rays, we solve the inverse problem allowing us to determine the appropriate wavefront phase law hence the phase function $\Phi(y)$ to be applied upon the phase modulator.

Fig.2 (a) and (b) are results of our approach applied to the well known case of the parabolic accelerating beam. The intensity profile along $y$ for a fixed $x$ is the desired Airy function modulated by a Gaussian envelope what validates our approach. The technique is readily generalizable, allowing convenient phasemask design for other profiles and Fig 2. (c) and (d) show, as an example, how a hyperbolic acceleration profile can be generated using such an approach. We anticipate that this novel inverse problem approach will allow a significant extension to techniques for the generation of tailored acceleration profiles for diverse applications in photonics.

\section{References}

[1] M. V. Berry,“Nonspreading wave packets,” Am. J. of Phys. 47, 264, (1979)

[2] G. A.Siviloglou, J.Broky, A. Dogariu, and D. N. Christodoulides, "Observation of Accelerating Airy Beams," Phys. Rev. Lett. 99, 213901, (2007)

[3] J. Baumgartl, M. Mazilu, and K. Dholakia, “Optically mediated particle clearing using Airy wavepackets,” Nat. Phot. 2, 675-678 (2008).

[4] D. G. Papazoglou, S. Suntsov, D. Abdollahpour, and S. Tzortzakis, "Tunable intense Airy beams and tailored femtosecond laser filaments," Phys. Rev. A 81, 061807, (2010)

[5] P. Polynkin, M. Kolesik, J. V. Moloney, G. A. Siviloglou,and D. N. Christodoulides, "Curved Plasma Channel Generation Using Ultraintense Airy Beams," Science 324, 229 (2009). 